\title{
Analysis of Fracture Behaviour of Exploded Metal Cylinders with Varied Charge
}

\author{
Xinlong Dong ${ }^{1}$, Xinlu $\mathrm{Yu}^{2}$ and Shunjie $\mathrm{Pan}^{1}$ \\ ${ }^{1}$ Faculty of Mechanical Engineering and Mechanics, Ningbo University, 315211, China \\ ${ }^{2}$ School of Mechatronical Engineering, Beijing Institute of Technology, Beijing, 10081, China
}

\begin{abstract}
Explosively driven fragmentation of ductile metals cylinders is a highly complex phenomenon. In this work, the fracture characteristics of exploded TA2 titanium alloy cylinder with varied charge were investigated numerically and experimentally. The results show that the fracture surfaces of fragments lie along planes of maximum shear stress for either a higher or a lower detonation pressure, but their mechanism is different. The finite element analysis demonstrated that the equivalent plastic strain in the middle of the wall is always larger than that of inner and outer wall for metal cylinder during the stage of shock wave driven period. For the high explosive pressures, the micro-cracks originated firstly in middle zone of wall during the stage of shock wave driven, and extend to the inner and outer wall in the direction of maximum shear stress. Explosives which generate lower detonation pressures, the shear fracture of cylinder originated from the inner wall and propagate to the outer wall in an angle of $45^{\circ}$ or $135^{\circ}$ to radial, the crack begin at the stage of free expansion. The simulated analysis of the process of deformation and fragmentation for exploded metal cylinder agree with the experimental results.
\end{abstract}

\section{Introduction}

The fragmentation of explosively driven cylinder has long been of interest in the military field, which relate to the applications including the design of fragment and blast resistant structures and protective facilities [1-4]. Gurney derive an empirical expression widely used in prediction of fragment velocities, which based on the principle of energy balance but do not concern the failure mechanism [1]. Taylor [3] developed a model to predict the radius with fracture based on the assumption that fracture would be radical. Mott developed models for prediction of fragment size concerning probability statement and release wave propagating from radial fracture surfaces [2]. These works laid the foundation of the study that continues to this day [4-6].

However, it is a highly complex phenomenon in which the fragmenting material is plastically deformed by the passage of an intense shock $\left(\sim 10^{2} \mathrm{GPa}\right)$ followed by high-rate expansion deformation that ultimately leads to fracture, more attention has been paid to the process and mechanism of fragmentation evolution in recent years[4-10]. Those works shown that several failure modes in the fragment cross-section could be occurs, including radial fracture, shear fracture, internal micro cracks, which are related to the cylindrical geometry, materials behaviour and loading characteristic of the experiment [4-9]. Hoggatt [4] illustrates that the characteristic of fracture modes observed when casings are fragmented by explosives were affected by stress state and thermo-plasticity. The shear fractures are observed when casings are fragmented by explosives having high detonation pressures, due to plastically extruding material in the inner region under triaxial compressive stress, the radial cracks appear firstly at the outer surface and propagate into the direction of maximum shear. The radial fractures are typical only when detonation pressures are relatively low, in which do not produce compressive hoop stresses over as large a portion of the wall, fragments are observed to possess radial fracture surfaces.

In this study, the fracture characteristics of exploded TA2 titanium alloy cylinder with varied charge were investigated numerically and experimentally. The study focuses on the process and mechanism of fragmentation of cylinder under different explosive pressure.

\section{FEM model of exploded cylinder}

A Finite element simulation was carried out using a hydro-code ABQUS to analysis the deformation process and fragmentation of the cylinder driven by explosive detonation. The two-dimensional FEM model is adapted as seen in Fig. 1. The element mesh size is about $50 \mu \mathrm{m}$ x $50 \mu \mathrm{m}$. Explosive loading was described by the radial shock loading $P(t)$ applied on inner surface of cylinder shell,

$$
p=p_{0} e^{-\left(t-t_{d}\right) / t_{d}}
$$

here, $P_{0}$ is Chapman-Jouguet pressure, $t_{d}$ is decay time.

\footnotetext{
Corresponding author: dongxinlong@nbu.edu.cn
} 


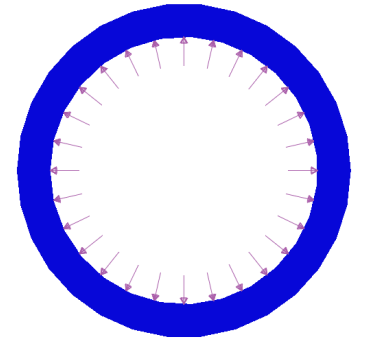

Fig. 1. FEM model.

The Grüneisen state equation and Johnson-Cook constitutive model are used for TA2 Titanium Alloy. The cohesive model for material failure is introduced, which is characterized by the progressive degradation of the material stiffness,

$$
D=\frac{\varepsilon-\varepsilon_{c r}}{\varepsilon_{f}-\varepsilon_{c r}} \quad \varepsilon_{c r}<\varepsilon<\varepsilon_{f}
$$

Here, $\varepsilon_{c r}$ critical plastic strain of damage initiation, $\varepsilon_{f}$ is fracture strain of the material. Once the initial of damage, the flow stress is,

$$
\sigma=\sigma_{0}(1-D)
$$

When $\mathrm{D}=0.9$, the material is no longer allowed to carry hydrostatic tension or deviator stresses.

\section{Simulation results}

Numerical simulations were performed for different charged condition examining the expanding deformation, stresses, strains and fracture of the cylinders.

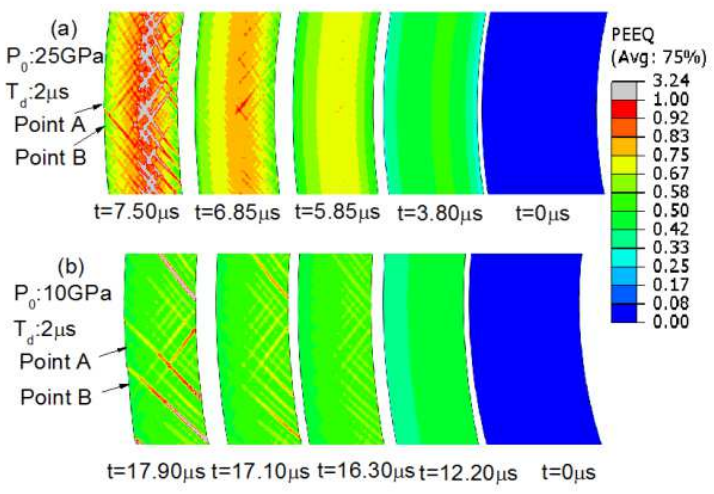

Fig.2. Evolution of equivalent plastic strain (eps).

Fig. 2(a), (b) represents typical examples of numerical simulations. The former example (a) shows numerical of equivalent plastic strain (eps) in the wall for cylinder charged with a strong explosive pulses $\left(\mathrm{P}_{0}=25 \mathrm{GPa}, \mathrm{t}_{\mathrm{d}}=2 \mu \mathrm{s}\right)$. It is seen that the eps in the middle of wall increases gradually, where the stain localization and micro cracks occur firstly. The strain localization or crack propagates to the inner and outer surfaces of wall at an angle of about $45^{\circ}$ or $135^{\circ}$ to radial. A feature of shear fracture is presented in casing fragmentation. The latter example (b) shows numerical eps evolution for cylinder subject to a lower explosive pulses $\left(\mathrm{P}_{0}=10 \mathrm{GPa}, \mathrm{t}_{\mathrm{d}}=2 \mu \mathrm{s}\right)$. Different from the cylinder fracture charged with higher pressure, the strain localization and cracks initialization at the inner wall and extend to outer surface at an angle of about $45^{\circ}$ or $135^{\circ}$ to radial. It is remarkable that, although both of the fractographic present the similar characteristics which are inclined at $45^{\circ}$ degrees respect to radial, the failure mechanism and process is different.

The simulation velocity history of the outer surface of cylinder with two different detonation pulses is shown in Fig. 3. The velocity is oscillations as the shock wave reflected back and forth between the inner and outer surfaces when exploded pulse loaded, and then retard the outward expansion of the cylinder in free expansion stage. From the results with two different pulse loading, we see that the cylinder break-up occur at $7.5 \mu$ s interval in stages of shock wave loading for a higher detonation pressure $\left(\mathrm{P}_{0}=25 \mathrm{GPa}\right)$, but for a lower detonation pressure $\left(\mathrm{P}_{0}=10 \mathrm{GPa}\right)$ the cylinder fracture at $17.90 \mu \mathrm{s}$ in the stage of cylinder free expansion.

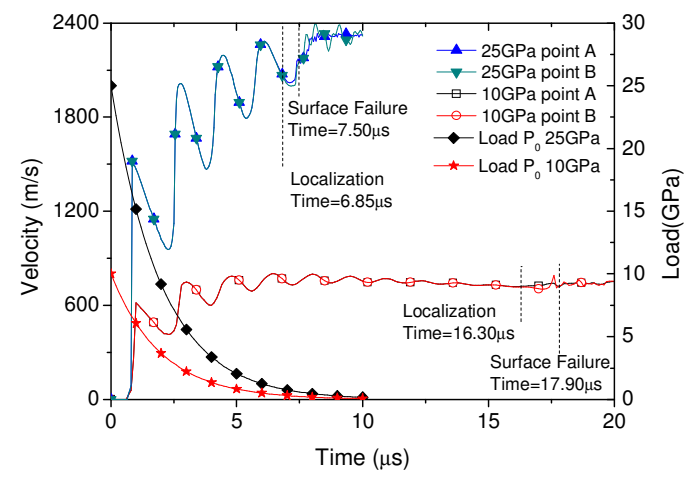

Fig.3. Calculated cylinder expansion velocity for different detonation pressures.

Fig. 4 shows numerical time-histories of triaxiality of stresses at inner surface, mid-thickness and outer surface. It is indicated that inner wall and outer wall are in negative and positive triaxiality respectively, while the triaxiality $\eta$ of mid-thickness cyclical changes in the state of tensile and compression with the shock wave reflected back and forth between the wall surfaces.

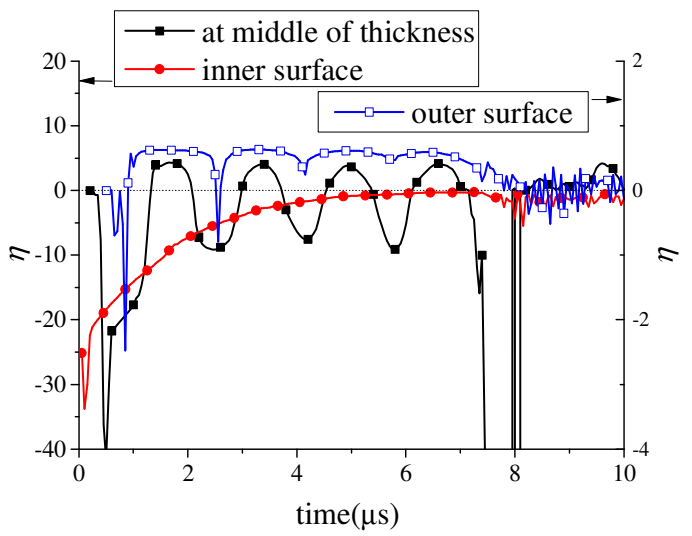

Fig. 4. Triaxiality of stress at three locations in wall. Positive triaxiality denotesa tensile stress state.

Figure 5 shows the stress component in the wall. The $\sigma_{\theta}, \sigma_{r}$ and $\sigma_{z}$ are circumferential stress, radial stress and 
axial stress in cylinder cross section. The results indicate that the hoop stress $\sigma_{\theta}$ of the outer wall is always in a state of tensile stress, but far less than that of midthickness. In general, tensile fracture could not origin firstly at the outer surface for a homogeneous metal tube. Figure 6 show the development of the equivalent plastic strain in wall. The equivalent plastic strain gradually decreasing from the inner surface to the outer wall of cylinder at initial expansion which stress wave first propagate from inner to outer wall (in the first stage shown in Fig. 6). Once the incident stress wave reaching the outer surface of cylinder followed reflecting back as a reversed elastic wave, the interaction of reflected unloading wave and incident stress wave could result in second-plastic zone in which the equivalent stress may exceeds the value that had ever reached and cause reverse loading [11]. Consequently, the equivalent plastic strain in mid-thickness become relatively larger than the inner and outer surface and the distribution of equivalent plastic strain in wall is into convex curve as seen the second stage shown in fig. 6. In this case, the initiation of strain localization and micro-crack would be expected at the mid-thickness of tube wall once the eps reached the critical damage and fracture strain of materials (seen third stage in Fig.6), then the crack developed to inner and outer wall in direction of maximum shear stress. This mode of failure is illustrated by Fig. 2(a).

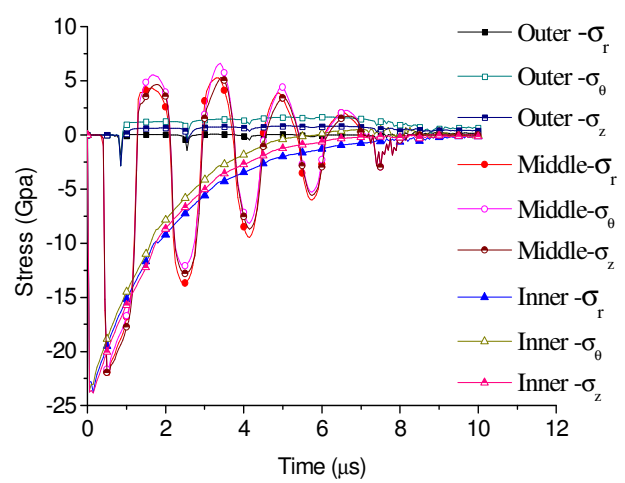

Fig. 5. Stress component in wall.

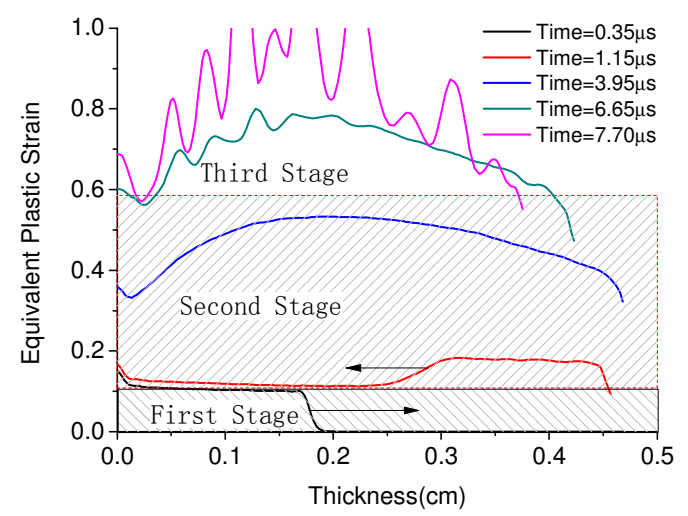

Fig. 6. Development of eps under high pressure.

Fig. 7 show development of equivalent plastic strain (eps) in cylinder wall using an explosive which has a lower detonation pressure $\left(\mathrm{P}_{0}=10 \mathrm{GPa}\right)$. Although the convex distribution of equivalent plastic strain in wall is similar to that of higher detonation pressure (Fig. 6), it does not result in stain localization or damage in midthickness due to the maximum value of equivalent plastic strain was less than the critical damage strain in explosive loading stage. Consequently, strain localization and fracture cracks would be initiated at inner wall and develop to outer surface of tube in stage of free expansion of cylinder (see Fig. 7). This mode of failure is illustrated by Fig. 2(b).

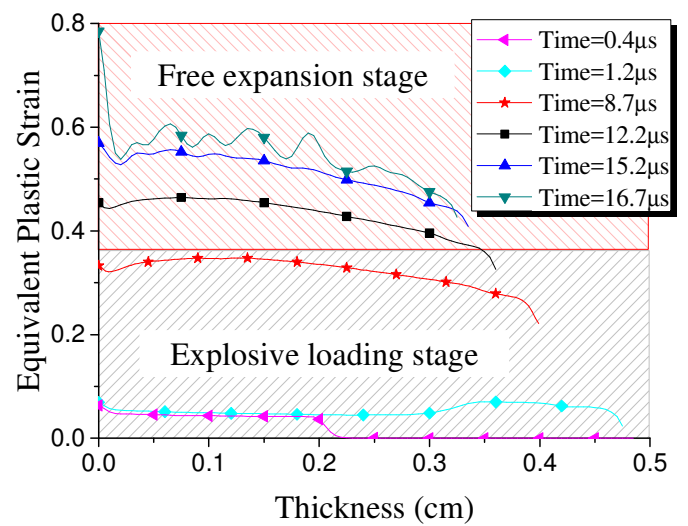

Fig. 7. Development of eps under lower pressure.

The results of finite element analysis demonstrated that the equivalent plastic strain in the middle of the wall is always larger than that of inner and outer wall for metal cylinder during the stage of expansion driven by detonation shock, where the secondary plastic zone was formed due to effect of shock wave reflection back and forth between inner and outer free surface. For the high explosive pressures, the micro-crack originated first in the secondary plastic zone during the stage of shock wave driven period, and then extended to the inner and outer wall in the direction of maximum shear, which is about $45^{\circ}$ or $135^{\circ}$ to the radial. Explosives which generate lower detonation pressures, the shear fracture of cylinder originated from the inner wall and propagates to the outer wall in an angle of $45^{\circ}$ or $135^{\circ}$, different from that of higher detonation pressure, the crack begin at the stage of free expansion. Although, the fragmentation for both higher and lower detonation pressure were all in shear fracture feature, their failure mechanisms are different.

\section{Analysis and discussion}

\subsection{Some problems}

It is generally accepted that the characteristic of primary radial cracks fractures occurred when metal cylinder are fragmented by explosives having lower detonation $[4,9$, 13]. The radial cracks origin at the outer surface and develop to the inner region due to tensile hoop stresses over as large a portion of the wall, and fragments are presented to possess radial fracture surfaces, the shear lips confined to only small region near inner of the wall [4]. However, the characteristic of primary shear fractures occurred when metal cylinder are fragmented 
by explosives having high detonation. In the case, small radial cracks which first appear at the outer surface, propagate in the direction of maximum shear due to the triaxial compression state extending from the internal radius to almost radius which depends upon internal pressure and initial tube dimensions [4]. Furthermore, adiabatic shear bands can be observed on fragments due to the thermal softening of material $[8,9,12]$. Recently, many experimental feature of deformation and fragmentation of expanding cylinders recorded by high speed cameras show that the time of visible crack to the outside surface of the cylinder is earlier apparent the time of smoke leaking through the cracks $[7,8,9,12]$. It is indicated apparently that the crack origin from the outer wall.

However, the results of finite element analysis demonstrated that, different from cylinder expanding under quasi-static conditions, neither the hoop stress nor equivalent plastic strain of outer surface are always less than that of the middle of the wall for exploded metal cylinder. It is generally impossible to appear failure firstly according to the rule of maximum stress or strain. Reference [11] showed similar results, they compared the analysis of finite element and experimental feature and resumed that the radial fracture begun outer wall might not be possible to happen for homogeneous materials, which might be affected by imperfections of the material or geometry of cylinder.

\subsection{Comparison with experimental results}

The experiments were designed to investigate the fracture characteristics of the metal cylinder that are explosively in higher and lower detonation pressures [14]. The cylinders material used is fabricated from TA2 titanium alloy, which components are Ti:99.6, C:0.021, Fe:0.06, N:0.025, H:0.004, O:0.11, with microstructure of $\alpha$ equal axis crystal. The cylinder has an outer diameter of $30 \mathrm{~mm}$ and with wall thickness $2.5 \mathrm{~mm}$. The length of cylinder used is $100 \mathrm{~mm}$.
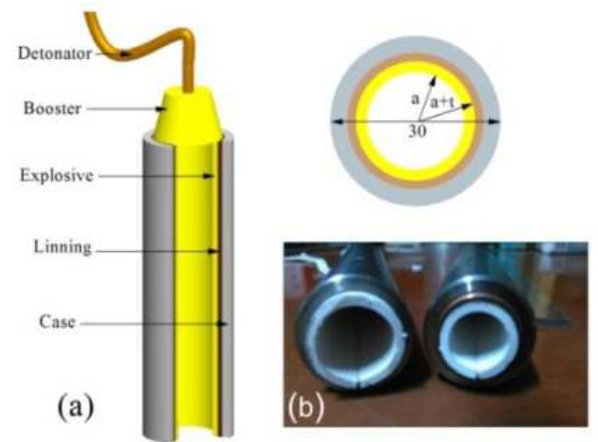

Fig. 8. Experiment set-up. (a) Schematics of a cylinders charged, (b) photo of specimen and charge.

The schematic representation of experimental setup for cylinder expansion test is shown in Fig. 8. The cylinder was filled with a hollow column explosive comprising a mixture of $80 \%$ PETN and additive, which is with density of $1.46 \mathrm{~g} / \mathrm{cm}^{3}$, the detonation velocity of about $8000 \mathrm{~m} / \mathrm{s}$. In order to investigate fracture characteristics of cylinder under different detonation pressure, TA2 titanium alloy cylinder test was carried out with two types of charge assembly. One PETN hollow columns filled the TA2 titanium alloy tubes, but, the other charged smaller diameter changes and same wall thickness were provided. In latter cases, pure copper sleeves (the wall thickness $\mathrm{t}=1.9 \mathrm{~mm}$.) are placed between the explosive and the cylinder wall, as shown in Fig. 7(a), (b). The purpose of this charge assembly is to provide a different detonation peak pressure. The exploding cylinder was modelled with hydrodynamics code LS-DYNA simulations allow us to evaluate detonation wave applied the cylinder. The simulations indicate loading pressure characteristic applied on the inner surface of the TA2 titanium alloy cylinder under different charge assembly, as shown in Fig. 9.

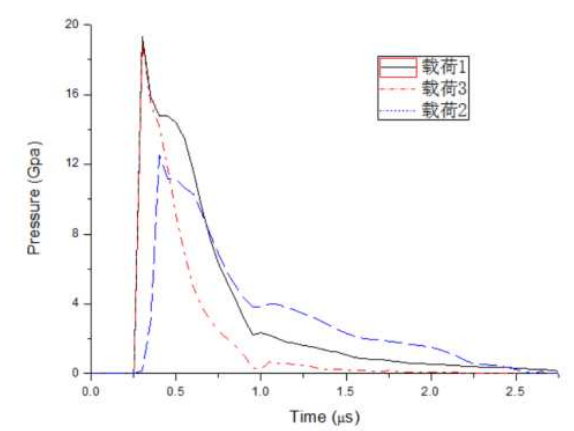

Fig. 9. Pressure-time curve with different charge assemblies.

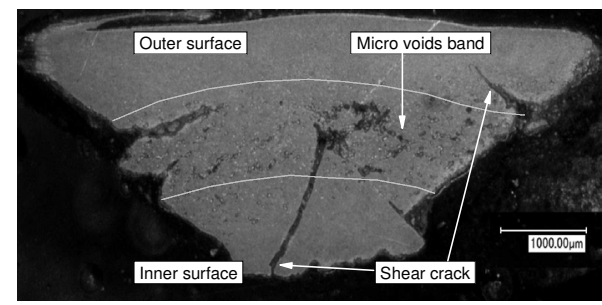

(a)

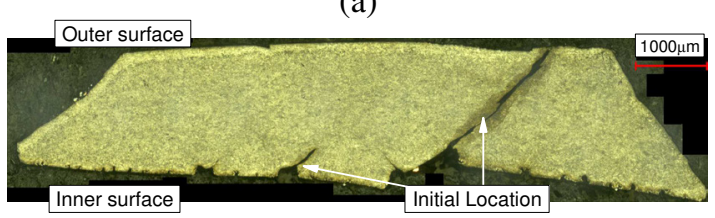

(b)

Fig. 10. Cross-section micrograph of fragment illustrating, (a) shear crack and voiding when subject to higher pressure, (b) shear fracture from inner-wall when subject to lower pressure.

The microscopic metallographic analysis of the recovered fragments was carried out. Fig. 10 gave the typical metallography of the cross- section of fragments. Fracture surfaces of fragments all lie along planes of maximum shear stress for TA2 titanium alloy cylinder exploded by either a higher or a lower detonation pressure, show the characteristics of shearing fracture. The sequence of photographs displayed in Fig. 3(a) clearly illustrates that the shearing fracture of TA2 titanium alloy cylinder driven by a high explosive pressure is initiated from the middle of the wall where a region of damage and micro-voids is formed firstly, then extends to inner and outer wall in an angle of about $45^{\circ}$ or $135^{\circ}$ to the radial. However, the cracks originate from 
the inner wall and extend to outer wall in an angle of $45^{\circ}$ or $135^{\circ}$ to the radial. The experimental phenomenon is similar to the FEM results, verified that the simulated analysis of the process of deformation and failure for exploded metal cylinder might be occurred.

It should be noted that the fragmentation characteristics of exploded metal cylinders do not only affected by the explosive pressure but also related to the material, configuration and other factors [7, 8, 10]. Goto [7] compared the fracture characteristics of cylinder made from AerMet 100 and AISI 1018. The two materials are significantly different in their microstructural morphologies and properties. The materials fail in different ways as well, in which the distributed micro-voids could be seen on the metallography of fragments for AISI 1018 steel, similar to that the feature of TA2 fragments in this paper.

\section{Conclusions}

In this work, the fracture characteristics of exploded TA2 titanium alloy cylinder with varied charge were investigated numerically and experimentally. The study focuses on the process and mechanism of fragmentation of cylinder under different explosive pressure. The results show the fracture surfaces of fragments present almost the feature of shearing fracture, lie along planes of maximum shear stress for either a higher or a lower detonation pressure, their failure mechanism is different.

The results of finite element analysis demonstrated that the equivalent plastic strain in the middle of the wall is always larger than that of inner and outer wall for metal cylinder during the stage of expansion driven by detonation shock. For the high explosive pressures, the micro-cracks originated first in the secondary plastic zone during the stage of shock wave driven period, and extend to the inner and outer wall in the direction of maximum shear. Explosives which generate lower detonation pressures, the shear fracture of cylinder originated from the inner wall and propagated to the outer wall in an angle of $45^{\circ}$ or $135^{\circ}$ to radial, the crack begin at the stage of free expansion.

The experimental results are similar to the FEM results, verified that the simulated analysis of the process of deformation and failure for exploded metal cylinder might be occurred.

The authors greatly appreciate the financial support from the National Science Foundation of China (No. 11672143, U1230122).

\section{References}

1. R W. Gurney, The initial velocities of fragments from bombs, shell and grenades, Army Ballistic Research Lab. Aberdeen Proving Ground MD (1943)

2. NF. Mott, Fragmentation of shell cases. Proc Royal Soc Lond A, 189:300-8 (1947)

3. G.I. Taylor, The fragmentation of tubular bombs. In: Scientific papers of G.I. Taylor, vol. III. Cambridge: Cambridge University Press. No. 44, pp. 387-390 (1963)

4. R.H. Hoggatt, R.F. Recht, Fracture behavior of tubular bombs. J Appl Phys, 39(3):1856-62 (1968)

5. D.E. Grady, M.M. Hightower, Natural Fragmentation of Exploding Cylinders, in Shock wave and high-strain-rate phenomena in material, Marcel Dekker Inc., pp.713-721 (1992)

6. M. Singh, H. R. Suneja, M. S. Bola, et al. Dynamic tensile deformation and fracture of metal cylinders at high strain rates, International journal of impact engineering, 27(9): 939-954 (2002)

7. D.M. Goto, R. Becker, T.J. Orzechowski et al, Investigation of the fracture and fragmentation of explosively driven rings and cylinders[J]. International Journal of Impact Engineering, 35(12): 1547-1556 (2008)

8. Haibo HU, Tiegang TANG, Bayi Hu et al, An study of uniform shear bands orientation selection tendency on explosively loaded cylinder shells, Explosion and Shock Waves, 24(2): 97-107 (2004)

9. Tiegang TANG, Qingzhong LI, Xuelin SUN, Strainrate effects of expanding fracture of 45 steel cylinder shells driven by detonation. Explosion and Shock Waves, 26(2): 129-133 (2006)

10. Shiwen Zhang, Shan Jin, Cangli Liu. Simulation of the dynamic expanding process of thick-walled cylinder with defects, Chinese Journal of Applied Mechanics, 27(3): 622-625 (2010)

11. Lili WANG, Foundation of Stress Waves, Beijing National Defense Press (2005)

12. Xinlu YU. Research on Shear Fracture Mechanism of Cylindrical Shells Subjected to Explosive Loading[D], Ningbo University (2014)

13. T. Hiroe, K. Fujiwara, H. Hata, H. Takahashi, Deformation and fragmentation behaviour of exploded metal cylinders and the effects of wall materials, configuration, explosive energy and initiated locations, International Journal of Impact Engineering, 35: 1578-1586 (2008)

14. Sunjie Pan, Xinlu Yu, Xinlong Dong, Experimental study of fragmentation behaviour of exploded TA2 alloy cylinder with varied charge, 31(4): 382-388 (2017) 
